\title{
LOCAL STRESSES AND STRAINS IN AXIALLY CRACKED CYLINDRICAL SHELLS
}

\author{
C.T. LIU and X.J. WU \\ Institute of Mechanics, Academia Sinica, Beijing, People's Republic of China
}

\begin{abstract}
A perturbation solution is obtained for the local stress-strain fields in an axially cracked cylindrical shell. The tenth-order differential equations are used that take into account the transverse shear deformation. The perturbation of a curvature parameter, $\lambda$, is employed, where $\lambda^{2}=\left[12\left(1-\nu^{2}\right)\right]^{1 / 2} a^{2} / R h$. The stress intensity factors for finite size cylindrical shells subjected to bending and internal pressure are evaluated. Sufficient accuracy can be obtained without using fine mesh sizes in regions near the crack tip. Also analyzed are the influence of cylinder diameter and shearing stiffness on bulging.
\end{abstract}

\section{Introduction}

The study of cracked cylindrical shell is of great importance to the safety of engineering structures such as pressure vessels and pipelines. This problem was investigated first by the classical shallow thin shell theory [1,2], then by Reissner's theory [3], where the integral equation approach was used. Since the problem is complex, only the local stresses were found to yield the stress intensity factors while the shell diameter effect is neglected. In [4], the quarter-point thick-shell elements were applied to cylindrical shells with cracks. But these elements can only approximate the stress fields in a region near the crack tip. In order to calculate stress intensity factors, it is necessary to use a more general solution for the crack tip stress-strain fields.

In this paper, a perturbation method is employed to solve the tenth-order differential equations. The configuration of a cracked spherical shell was solved analytically in [5] while the case of a shallow thin spherical shell with cracks is treated in [6]. Perturbation is performed using the curvature parameter, $\lambda$. In addition to including the transverse shear deformation, a special 8-node thick-shell element is used to obtain the local stresses and displacements. Numerical results are obtained for problems symmetric with reference to the crack. The effects of the size of cylinder on the crack tip stress intensity factors are also discussed.

\section{Governing equations}

A cylindrical shell of radius $R$ and thickness $h$, containing an axial through crack of length $2 a$, is shown in Fig. 1. The crack tip coincides with the origin of the coordinates. The governing equations for cylindrical shells with shear deformation are well known:

$$
\begin{aligned}
& D\left(\frac{\partial^{2} \psi_{x}}{\partial x^{2}}+\frac{1-\nu}{2} \frac{\partial^{2} \psi_{x}}{\partial y^{2}}+\frac{1+\nu}{2} \frac{\partial^{2} \psi_{y}}{\partial x \partial y}\right)+C\left(\frac{\partial w}{\partial x}-\psi_{x}\right)=0, \\
& D\left(\frac{\partial^{2} \psi_{y}}{\partial y^{2}}+\frac{1-\nu}{2} \frac{\partial^{2} \psi_{y}}{\partial x^{2}}+\frac{1+\nu}{2} \frac{\partial^{2} \psi_{x}}{\partial x \partial y}\right)+C\left(\frac{\partial w}{\partial y}-\psi_{y}\right)=0, \\
& C\left(\nabla^{2} w-\frac{\partial \psi_{x}}{\partial x}-\frac{\partial \psi_{y}}{\partial y}\right)+\frac{1}{R} \frac{\partial^{2} \phi}{\partial x^{2}}+P=0, \\
& \frac{1}{B} \nabla^{2} \nabla^{2} \phi+\frac{1}{R} \frac{\partial^{2} w}{\partial x^{2}}=0,
\end{aligned}
$$




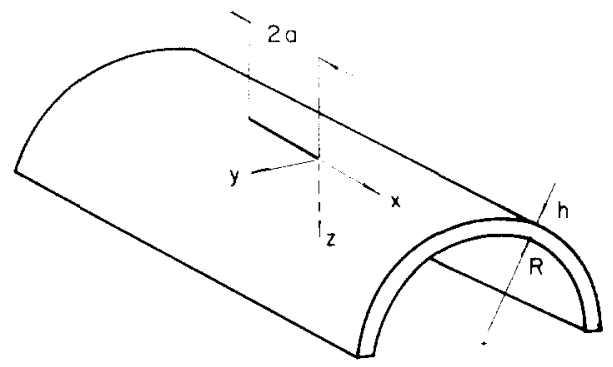

Fig. 1. Cylindrical shell with axial crack.

in which $P$ is the internal pressure and $w$ is the transverse displacement. In eq. (1), $B$ is the extension-compression stiffness, $D$ the bending stiffness, and $C$ the shearing stiffness. The function $\phi$ is related to the resultant stresses $N_{x}, N_{y}$ and $N_{x y}$ as follows:

$$
N_{x}=\frac{\partial^{2} \phi}{\partial y^{2}}, \quad N_{y}=\frac{\partial^{2} \phi}{\partial x^{2}}, \quad N_{x, y}=-\frac{\partial^{2} \phi}{\partial x \partial y} .
$$

The transverse shear $\psi_{x}$ and $\psi_{y}$ can be expressed in terms of the displacement functions $F$ and $f$ to simplify the governing equations. Application of

$$
\psi_{x}=\frac{\partial F}{\partial x}+\frac{\partial f}{\partial y}, \quad \psi_{y}=\frac{\partial F}{\partial y}-\frac{\partial f}{\partial x}
$$

to eqs. (1), results in:

$$
\begin{array}{ll}
\nabla^{2} \nabla^{2} \psi+\lambda^{2} \frac{\partial^{2} w}{\partial x^{2}}=0 & \nabla^{2} \nabla^{2} F-\lambda^{2} \frac{\partial^{2} \psi}{\partial x^{2}}=0, \\
w=\left(1-\kappa \nabla^{2}\right) F+\operatorname{Im} \Phi, & \nabla^{2} f-4 \mu^{2} f=4 \mu^{2} \operatorname{Re} \Phi,
\end{array}
$$

in which $\Phi$ is an analytic function and

$$
\psi=\frac{\phi}{\sqrt{B D}}, \quad \lambda^{2}=\frac{\sqrt{12\left(1-\nu^{2}\right)}}{R h} a^{2}, \quad \kappa=\frac{D}{C a^{2}}, \quad 2 \mu^{2}=\frac{1}{(1-\nu) \kappa} .
$$

\subsection{Power-series expansion}

Now, expand $\psi, F$, and $w$ in power series of $\lambda^{2}$, i.e.

$$
\psi=\sum_{n=0}^{\infty} \lambda^{2 n} \psi_{n}, \quad F=\sum_{n=0}^{\infty} \lambda^{2 n} F_{n}, \quad w=\sum_{n=0}^{\infty} \lambda^{2 n} w_{n}, \quad \Phi=\sum_{n=0}^{\infty} \lambda^{2 n} \Phi_{n}, \quad f=\sum_{n=0}^{\infty} \lambda^{2 n} f_{n} .
$$

Substituting eqs. (6) into eq. (4), the perturbation equations for $n=0$ take the form:

$$
\begin{array}{ll}
\nabla^{2} \nabla^{2} \psi_{0}=0, & \nabla^{2} \nabla^{2} F_{0}=0, \\
\boldsymbol{w}_{0}=\left(1-\kappa \nabla^{2}\right) F_{0}+\operatorname{Im} \Phi_{0}, & \nabla^{2} f_{0}-4 \mu^{2} f_{0}=4 \mu^{2} \operatorname{Re} \Phi_{0} .
\end{array}
$$

The governing equations for a flat plate [7] can thus be obtained. They are listed in the Appendix. 


\subsection{Boundary conditions}

For the present problem, the boundary conditions on the free crack surfaces are

$$
M_{y}=M_{x y}=Q_{y}=N_{y}=N_{x y}=0 \text { at } \theta= \pm \pi,
$$

where $\theta$ is measured counterclockwise from the $x$-axis. The quantities $M_{y}$ and $M_{x y}$ are the bending moments and $Q_{y}$ is the transverse shear force.

\section{Stress intensity factors}

Figure 2 shows the grid pattern for a cylindrical shell with an axial crack of half length $a$ along the $x$-axis. The half length of the shell is $L$. A special finite element is used to represent the stress and strain field at the crack tip. Other elements are the regular quadratic type for the thick shell. Poisson's boundary conditions in eq. (8) are satisfied.

Numerical results for the stress intensity factors are given for two loading conditions: the constant bending moment and the uniform internal pressure. The former refers to the bending moment $M_{0}$ with a maximum surface stress of $\sigma_{\mathrm{b}}=6 M_{0} / h^{2}$. The corresponding Mode I stress intensity factor is given by

$$
K_{\mathrm{I}}=\left(K_{\mathrm{mb}}+K_{\mathrm{bb}}\right) \sigma_{\mathrm{b}} \sqrt{\pi a} .
$$

For a uniform internal pressure $P_{0}$ with a membrane stress $\sigma_{\mathrm{m}}=P_{0} R / h$, the result is

$$
K_{\mathrm{I}}=\left(K_{\mathrm{mm}}+K_{\mathrm{bm}}\right) \sigma_{\mathrm{m}} \sqrt{\pi a} .
$$

In eqs. (9) and (10), $K_{\mathrm{mb}}$ and $K_{\mathrm{mm}}$ correspond to extension and $K_{\mathrm{bb}}$ and $K_{\mathrm{bm}}$ to bending.

\subsection{Bending of finite radius cylindrical shell}

A cylinder of length $2 L$ is subjected to a constant bending moment $M_{0}$. The results are given in Table 1. When $a / L=0.01$, the intensity factor $K_{\mathrm{I}}$ agrees well with that obtained in [3] for an infinite shell with a $2 \%$ deviation. When $a / L$ increases, both the extensional and bending stress intensity factors increase. Their variations are shown in Fig. 3, which also contains the result in [4] obtained by using quarter-point thick-shell elements. The error is about $14 \%$.

\subsection{Pressurized cylindrical shell}

Numerical results are also obtained for cylinders with a ratio of $a / L=0.03$. Figures 4 and 5 show, respectively, the effect of shearing stiffness on the extensional and bending stress intensity factors, $K_{\mathrm{mm}}$

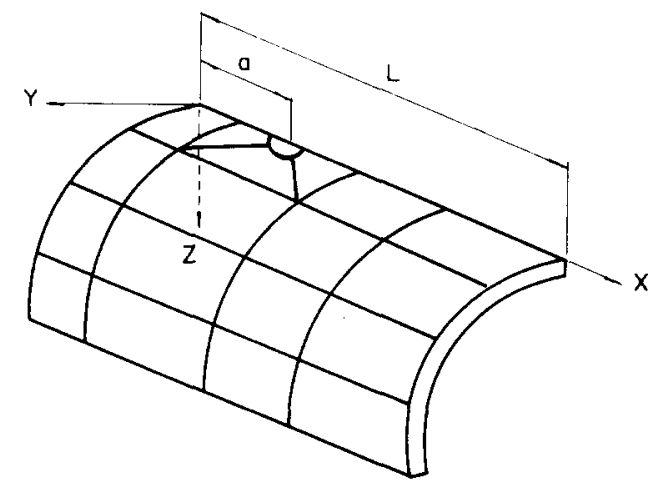

Fig. 2. Grid pattern for one-half crack and shell geometry.

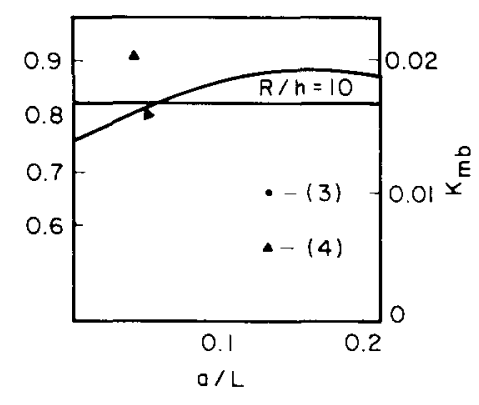

Fig. 3. Variations of $K_{\mathrm{bb}}$ and $K_{\mathrm{mb}}$ with $a / L$. 
Table 1

Stress intensity factors under bending

\begin{tabular}{llllll}
\hline & $a / L$ & $0[3]$ & 0.01 & 0.1 & 0.2 \\
\hline $\begin{array}{l}R / h=10 \\
\nu=0.25\end{array}$ & $K_{\mathrm{hb}}$ & 0.8320 & 0.8153 & 0.8202 & 0.8206 \\
& & & & 0.0189 & 0.0201 \\
\hline
\end{tabular}

Table 2

Bulging factor $M(a / L=0.03)$

\begin{tabular}{llllll}
\hline$\lambda$ & $\kappa$ & & & & 0.1 \\
\cline { 2 - 5 } & $0^{\mathrm{a}}$ & 0.001 & 0.05 & 1.2024 & 0.5 \\
\hline 0.5 & 1.0619 & 1.1640 & 1.1702 & 1.3480 & 1.4229 \\
1.0 & 1.2219 & 1.3052 & 1.2691 & 1.9333 & \\
2.0 & 1.7239 & 1.8376 & 1.7065 & & \\
3.0 & 2.2317 & 2.3452 & 2.2850 & & \\
4.0 & 2.9813 & 2.7916 & & & \\
\hline
\end{tabular}

a When $\kappa=0, M=\left(1+0.49 \lambda^{2}\right)^{1 / 2}$.

Table 3

Effect of cylinder radius constraint

\begin{tabular}{|c|c|c|c|}
\hline \multirow[t]{2}{*}{$\lambda$} & \multicolumn{3}{|l|}{$a / L$} \\
\hline & 0 & 0.03 & 0.2 \\
\hline 1.0 & 1.2219 & 1.3052 & 1.4137 \\
\hline 2.0 & 1.7239 & 1.8376 & 1.9305 \\
\hline 3.0 & 2.2317 & 2.3452 & 2.5284 \\
\hline 4.0 & 2.9813 & 2.7916 & 2.9923 \\
\hline
\end{tabular}

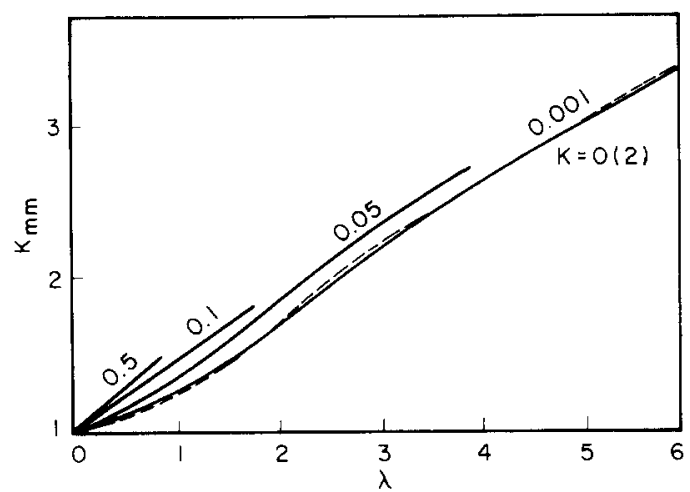

Fig. 4. Variations of $K_{\mathrm{mm}}$ with $\lambda$.

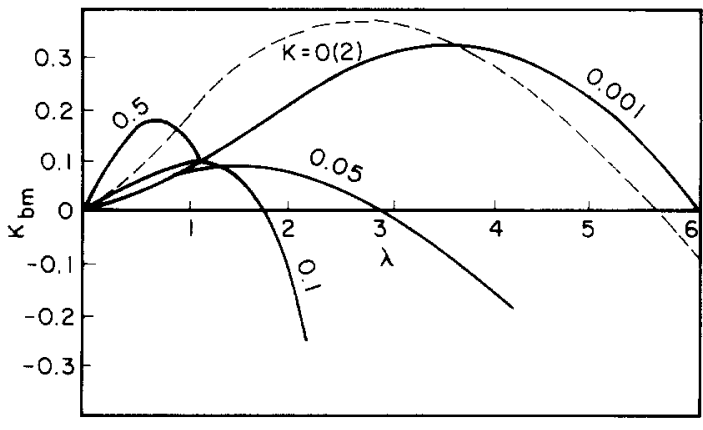

Fig. 5. Variations of $K_{\mathrm{bm}}$ with $\lambda$. 


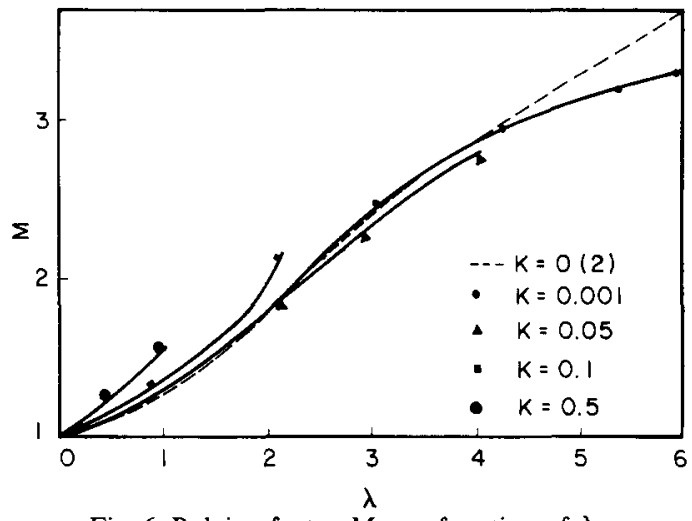

Fig. 6. Bulging factor $M$ as a function of $\lambda$.

and $K_{\mathrm{bm}}$. Note from Fig. 4 that $K_{\mathrm{mm}}$ approaches the classical limit when $\kappa \rightarrow 0$, but $K_{\mathrm{bm}}$ differs considerably from the thin-shell theory prediction.

Combining the factors $K_{\mathrm{mb}}$ and $K_{\mathrm{mm}}$ in eqs. (9) and (10), a bulging factor,

$$
M=K_{\mathrm{mm}}+K_{\mathrm{bm}},
$$

can be defined. Refer to Table 2 for values of $M$. Figure 6 shows the variations of $M$ with $\lambda$ for different $\kappa$. A maximum effect of $33 \%$ is found. Table 3 summarizes the effect of bulging due to $a / L$ for $\kappa=0.001$. For $a / L<0.2$, the maximum effect of cylinder radius is about $12 \%$. This deviation becomes larger as $\kappa$ increases.

\section{Conclusions}

A perturbation solution of local stress-strain fields for an axially cracked cylindrical shell is obtained using the tenth-order governing differential equation, where the Poisson's boundary conditions are satisfied instead of those of Kirchhoff for shallow thin shells.

Using a special crack tip element for thick shell, Mode I stress intensity factors are obtained for the cases of bending and pressurized load. Influences due to the constraint of the shell radius are exhibited. For small curvature, the present results agree well with those obtained previously. In particular, the extensional component of the stress intensity factor appears to be a lower limit of the Reissner's plate solution, while that of bending differed considerably from that of the present theory.

\section{Appendix. Flat-plate equations}

With reference to eq. (7), the following results pertain to the solution of a flat plate in [7]:

For $l=\frac{1}{2}, \frac{3}{2}, \frac{5}{2}, \ldots$, there results

$$
\begin{gathered}
\psi_{0}=\sum_{l} r^{l+1}\left\{A_{l}\left[\cos (l-1) \theta-\frac{l-1}{l+1} \cos (l+1) \theta\right]+B_{l}[\sin (l-1) \theta-\sin (l+1) \theta]\right\}, \\
F_{0}=\sum_{l} r^{l+1}\left\{K_{l}\left[\cos (l-1) \theta-\frac{4+(l-1)(1-\nu)}{(l+1)(1-\nu)} \cos (l+1) \theta\right]\right. \\
\left.+L_{l}\left[\sin (l-1) \theta+\frac{4-(l+1)(1-\nu)}{(l+1)(1-\nu)} \sin (l+1) \theta\right]\right\}
\end{gathered}
$$




$$
\begin{aligned}
f_{0}=\sum_{l} r^{l+1} \frac{\mu^{2}}{l}\{ & -4 \kappa l K_{l}[\sin (l-1) \theta-\sin (l+1) \theta] \\
& \left.+\left(\tilde{\beta}_{l-1}+4 \kappa l L\right)\left[\cos (l-1) \theta-\frac{l-1}{l+1} \cos (l+1) \theta\right]\right\} \\
+ & \sum_{l} \sum_{n, m=0}^{\infty}\left[-4 \kappa l K_{l} \sin (l-1+2 n) \theta+\frac{l-1}{l-1+2 n}\left(\tilde{\beta}_{l-1}+4 \kappa l L_{1}\right) \cos (l-1+2 n) \theta\right] \\
& \frac{(-1)^{n} \mu^{2(m+n)} r^{l-1+2(m+n)}}{n ! \phi(l+n-2, n) m ! \phi(l-1+2 n, m)}, \\
w_{0}= & \sum_{l} r^{l+1}\left\{K_{l}\left[\cos (l-1) \theta-\frac{4+(l-1)(1-\nu)}{(l+1)(1-\nu)} \cos (l+1) \theta\right]\right. \\
& +\sum_{l} r^{l-1} \tilde{\beta}_{l-1} \sin (l-1) \theta,
\end{aligned}
$$

where $m+n>1$ and $\tilde{\beta}_{l-1}=0$ for $l<\frac{3}{2}$.

For $l=1,2,3, \ldots$, the following applies:

$$
\begin{aligned}
& \psi_{0}=\sum_{l} r^{l+1}\left\{A_{l}[\cos (l-1) \theta-\cos (l+1) \theta]+B_{l}\left[\sin (l-1) \theta-\frac{l-1}{l+1} \sin (l+1) \theta\right]\right\} . \\
& F_{0}=\sum_{l} r^{l+1}\left\{K_{l}\left[\cos (l-1) \theta+\frac{4-(l+1)(l-\nu)}{(l+1)(1-\nu)} \cos (l+1) \theta\right]\right. \\
& \left.+L_{l}\left[\sin (l-1) \theta-\frac{4+(l-1)(1-\nu)}{(l+1)(1-\nu)} \sin (l+1) \theta\right]\right\}, \\
& f_{0}=\sum_{l} r^{l+1} \frac{\mu^{2}}{l}\left\{-\left(\tilde{\alpha}_{l-1}+4 \kappa l K_{l}\right)\left[\sin (l-1) \theta-\frac{l-1}{l+1} \sin (l+1) \theta\right]\right. \\
& \left.+4 K l L_{l}[\cos (l-1) \theta-\cos (l+1) \theta]\right\} \\
& +\sum_{l} \sum_{m, n=0}^{\infty}\left[-\frac{l-1}{l-1+2 n}\left(\tilde{\alpha}_{l-1}+4 \kappa l K_{l}\right) \sin (l-1+2 n) \theta\right] \\
& +4 \kappa l L_{l}[\cos (l-1+2 n) \theta] \frac{(-1)^{n} \mu^{2(m+n)} r^{l-1+2(m+n)}}{n ! \phi(l+n-2, n) m ! \phi(l-1+2 n, m)}, \\
& w_{0}=\sum_{l} r^{l+1}\left\{K_{l}\left[\cos (l-1) \theta+\frac{4-(l+1)(1-\nu)}{(l+1)(1-\nu)} \cos (l+1) \theta\right]\right. \\
& \left.+L_{l}\left[\sin (l-1) \theta-\frac{4+(l-1)(1-\nu)}{(l+1)(1-\nu)} \sin (l+1) \theta\right]\right\} \\
& +\sum_{l} r^{l-1} \tilde{\alpha}_{l-1} \cos (l-1) \theta,
\end{aligned}
$$

where $m+n>1$ and $\tilde{\boldsymbol{\alpha}}_{l-1}=0$ for $l<\frac{3}{2}$ and $\phi(l, m)=\Gamma(l+m+1) / \Gamma(l+1)=(l+1)(l+2) \ldots(l+m)$. 
The equations for $n \geq 1$ are given by:

$$
\begin{array}{ll}
\nabla^{2} \nabla^{2} \psi_{n}=-\frac{\partial^{2} w_{n-1}}{\partial x^{2}}, & \nabla^{2} \nabla^{2} F_{n}=\frac{\partial^{2} \psi_{n-1}}{\partial x^{2}}, \\
w_{n}=\left(1-\kappa \nabla^{2}\right) F_{n}+\operatorname{Im} \Phi_{n}, & \nabla^{2} f_{n}-4 \mu^{2} f_{n}=4 \mu^{2} \operatorname{Re} \Phi_{n} .
\end{array}
$$

The first-order solutions for $n=1$ and $l=\frac{1}{2}, \frac{3}{2}, \frac{5}{2}, \ldots$, are:

$$
\begin{gathered}
\begin{array}{c}
\psi_{1}=\sum_{l} r^{l+3}\left\{K _ { l } \left[-e_{1} \cos (l-3) \theta+\left(e_{2}+e_{3}\right) \cos (l-1) \theta\right.\right. \\
\left.+\frac{(l-3) e_{1}-(l-1)\left(e_{2}+e_{3}\right)}{l+1} \cos (l+1) \theta\right] \\
+L_{l}\left[-e_{1} \sin (l-1) \theta-\left(e_{2}-e_{4}\right) \sin (l-1) \theta\right. \\
\left.\left.+\left(e_{1}+e_{2}-e_{4}\right) \sin (l+1) \theta\right]\right\}
\end{array} \\
\quad-\sum_{l} r^{l+1} e_{5} \tilde{\beta}_{l-1}[\sin (l-3) \theta-\sin (l-1) \theta], \\
P_{1}=\sum_{l} r^{l+3}\left\{A_{l}\left[e_{1} \cos (l-3) \theta-e_{3} \cos (l-1) \theta+C_{1} \cos (l+1) \theta\right]\right. \\
\left.\quad+B_{l}\left[e_{1} \sin (l-3) \theta-e_{4} \sin (l-1) \theta+D_{1} \sin (l+1) \theta\right]\right\}, \\
f_{1}=\sum_{l} \sum_{n, m=0} A_{l} E_{l+1} \sin (l+1+2 n) \theta \cdot \frac{(-1)^{n} \mu^{2(m+n)} r^{l+1+2(m+n)}}{n ! \phi(l+n, n) m ! \phi(l+1+2 n, m)}, \\
\phi_{1}=-\sum_{l} r^{l+1} \mathrm{i} B_{l+1}[\cos (l+1) \theta+\mathrm{i} \sin (l+1) \theta],
\end{gathered}
$$

where $m+n>0$ and

$$
\begin{aligned}
& C_{1}=-\frac{[12 l+(1-\nu)(l+1)(l+2)](l-3) e_{1}-[8+(l+2)(1-\nu)](l-1)(l+1) e_{3}}{[4+(l+1)(1-\nu)](l+1)(l+2)}, \\
& D_{1}=-\frac{[12 l-(1-\nu)(l+2)(l+3)] e_{1}-[8(l+1)-(1-\nu)(l-3)(l+2)] e_{4}}{[4-(l+3)(1-\nu)](l+2)}, \\
& E_{l+1}=-4 \kappa(1-\nu) \frac{\left[2(l-3) e_{1}-l e_{3}\right](l-1)}{4+(l-1)(1-\nu)} .
\end{aligned}
$$

If $l=1,2,3, \ldots$, the following apply:

$$
\begin{gathered}
\psi_{1}=\sum_{l} r^{l+3}\left\{K_{l}\left[-e_{1} \cos (l-3) \theta-\left(e_{2}-e_{4}\right) \cos (l-1) \theta+\left(e_{1}+e_{2}-e_{4}\right) \cos (l+1) \theta\right]\right. \\
+L_{l}\left[-e_{1} \sin (l-1) \theta+\left(e_{2}+e_{3}\right) \sin (l-1) \theta\right. \\
\left.\left.\quad+\frac{(l-3) e_{1}-(l-1)\left(e_{2}+e_{3}\right)}{l+1} \sin (l+1) \theta\right]\right\} \\
+\sum_{l} r^{l+1} e_{5} \tilde{\alpha}_{l-1}[\cos (l-3) \theta-\cos (l-1) \theta],
\end{gathered}
$$




$$
\begin{aligned}
& P_{1}=\sum_{l} r^{l+3}\left\{A_{l}\left[e_{1} \cos (l-3) \theta-e_{4} \cos (l-1) \theta+C_{l} \cos (l+1) \theta\right]\right. \\
& \left.\quad+B_{l}\left[e_{1} \sin (l-3) \theta-e_{3} \sin (l-1) \theta+D_{1} \sin (l+1) \theta\right]\right\}, \\
& f_{1}=\sum_{l} \sum_{n, m=0}^{\infty} B_{l} F_{l+1} \cos (l+1+2 n) \theta \cdot \frac{(-1)^{n} \mu^{2(m+n)} r^{l+1+2(m+n)}}{n ! \phi(l+n, n) m ! \phi(l+1+2 n, m)}, \\
& \phi_{1}=\sum_{l} r^{l+1} F_{l+1}[\cos (l+1) \theta+\mathrm{i} \sin (l+1) \theta],
\end{aligned}
$$

where $m+n>0$ and

$$
\begin{aligned}
& C_{1}=-\frac{[12 l-(1-\nu)(l+2)(l+3)] e_{1}-[8(l+1)-(1-\nu)(l-3)(l+2)] e_{4}}{[4-(l+3)(1-\nu)](l+2)}, \\
& D_{1}=-\frac{[12 l+(1-\nu)(l+1)(l+2)](l-3) e_{1}-[8+(l+2)(1-\nu)](l-1)(l+1) e_{3}}{[4+(l+1)(1-\nu)](l+1)(l+2)}, \\
& F_{l+1}=-4 \kappa(1-\nu) \frac{\left[(l-3)(4 l+2) e_{1}+(l-1)(3 l+4) e_{3}\right]}{4+(l-1)(1-\nu)}
\end{aligned}
$$

The quantities $e_{j}(j=1, \ldots, 4)$ stand for

$$
\begin{aligned}
& e_{1}=l(l-1) / G(l-3), \quad e_{2}=4 l /(1-\nu) G(l-1), \quad e_{3}=l(l-3) / G(l-1), \\
& e_{4}=l(l-1) / G(l-1), \quad e_{5}=(l-1)(l-2) / H(l-3) ; \\
& G(l)=l^{4}-\left[(l+1)^{2}+(l+3)^{2}\right] l^{2}+(l+1)^{2}(l+3)^{2}, \\
& H(l)=l^{4}-\left[(l+1)^{2}-(l-1)^{2}\right] l^{2}+(l+1)^{2}(l-1)^{2} .
\end{aligned}
$$

\section{References}

[1] E.S. Folias, "An axial crack in a pressurized cylindrical shell", Int. J. Fracture Mech. I, 104-113 (1965).

[2] F. Erdogan and J. Kibler, "Cylindrical and spherical shells with cracks", Int. J. Fracture Mech. 5, 229-237 (1969).

[3] S. Krenk. "Influence of transverse shear on an axial crack in a cylindrical shell", Int. J. Fracture Mech. 14. 123-143 (1978).

[4] R.S. Barsoum, R.W. Loomis and B.D. Stewart. "Analysis of through cracks in cylindrical shells by the quarter-point elements", Int. J. Fracture Mech. 15, 259-280 (1979).

[5] G.C. Sih and H.C. Hagendorf, "A new theory of spherical shells with cracks", in: Thin-Shell Structures: Theory, Experiment and Design, Y.C. Fung and E.E. Sechler, eds., Prentice-Hall, New Jersey, 519-545 (1974).

[6] G.C. Sih and P.S. Dobreff, "Crack-like imperfections in a spherical shell”, Glasgow Math. J. 12, 104-113 (1971).

[7] C.T. Liu and Y.Z. Li, "The stress-strain fields at crack tip and stress intensity factors in Reissner's plate”, Proc. ICF Int. Symp. on Fract. Mech., Beijing (1983). 